\title{
The Study on Construction Scheme of Computer Laboratory Room Based on DAWNSTOR Desktop Virtualization System
}

\author{
Feng-Ying GUO ${ }^{1, a, *}$, Xing ZHAl, b ${ }^{1,}$ You-Liang HUANG ${ }^{1, c}$, \\ Jing-Zhong $\mathrm{LI}^{1, d}$, Xing-Guang MA ${ }^{1, e}$ \\ ${ }^{1}$ Information Center, Beijing University of Chinese Medicine, Beijing 100029, China \\ *aguofy@bucm.edu.cn, bzhaix@bucm.edu.cn, chuangyl@bucm.edu.cn, dlijz@bucm.edu.cn, \\ emxg@bucm.edu.cn, \\ ${ }^{*}$ Feng-Ying GUO
}

Keywords: desktop virtualization; DAWNSTOR; Computer laboratory room; construction scheme

\begin{abstract}
According to the problems about obsolete equipment, experimental environment less extendibility, complex maintenance management, the data stability security of the old traditional computer laboratory and other issues, this paper introduced technical features and architecture of desktop virtualization. Taking our school computer room construction as an example and to build a virtual cloud desktop computer room with the help of desktop virtualization technology, this paper described in detail virtual cloud desktop computer room construction plan based on the DAWNSTOR desktop virtualization system.
\end{abstract}

\section{Introduction}

With the wide use of computer in Universities, Some problems have been more and more attention to the management of all levels of the University, such as how to improve the management level of computer integrated application in Universities and how to make computer to provide more intimate features for users. Computer virtualization technology mainly includes server virtualization, application virtualization and desktop virtualization. Desktop Virtualization (sometimes called client virtualization) can separate desktop environments from physical machines into client - server computing models.

At present, Beijing University of Chinese Medicine (hereinafter referred to as "my university") has three campuses. The computer laboratory are distributed over a number of teaching upstairs in each campus. Thus, the computer laboratory management often encounter a lot of problems.

(1) The large workload of management and maintenance about System and software version update (2) Hardware information can't be fully understood in real time. Thus, computer laboratory administrator unable to control access to external devices and its security hidden danger (3) Computer laboratory administrator can't centrally manage and control the computers, and can't monitor the fault in real time and solve it remotely. (4) Obsolete equipment and long-term use of the reduction card lead to slow running. (5) When meeting the special needs (such as Chinese examination, the physician qualification examination), switching system requires a lot of manpower and time.

Therefore, the author unified configuration and management of all computer desktop and application with Method of desktop virtualization. The purpose is to improve the management and application of computer laboratory after the implementation of the desktop virtualization project, to make the Managers free from the tedious repetitive work with running in the teaching building and the campus. The development and research based on desktop virtualization system can not only effectively reduce the burden of computer laboratory computer management staff and uniform management of computer laboratory, but also play the performance of the computer itself to provide personalized desktop services with high performance for teachers and students. Which has a very important role in the management of the laboratory. 


\section{The Construction Scheme of Our University Computer Virtual Laboratory Base on DAWNSTOR}

The DAWNSTOR soft use desktop Virtualization Management Technology of independent copyright of Beijing morning overflow of the sea company. Which can be able to meet the needs of multi-system, multi-application and personalized teaching, and can realize the unified management and stable operation of the teaching environment, test environment, learning environment and open experimental environment. The underlying mechanism with background centralized control is adopted in the software. It can effectively prevent the client from virus damage and operation damage. And it has other functions. Such as supporting the rapid deployment of the system environment, supporting VLAN and cross routing management, supporting multi-brand client's unified mirror image, supporting the normal operation of broken network, the client server downtime, and supporting online update. The maintenance and upgrading of the background and other operations do not affect the normal use of the client.

\section{The Overall Objectives of the Construction Scheme}

\section{Push Mechanism on Demand}

There are many application system images on the virtual server. When the client is turned on, the server will distributes private application system and desktop for client according to the client's landing the different accounts. It can push their individual needs for each class, each student.

\section{The Virtual Local Caching Mechanism}

For the new computer, it can be a naked computer without formatting the hard disk before using. For the first time, the server will automatically push the system data on the server to the client hard disk using ON-Demand technology. Client hard disk can store user data and system temporary files and so as to form a cache. When the user needs to use more data in the process of using the computer, the server will send the corresponding data to the client's hard disk. The client hard disk will form a complete read and write cache, by marking all the data from the server and users in the use of self-created data. Every time the client starts later, the DAWNSTOR server only transmits a small amount of I/O read and write data. This part of the data and the data in the cache is combined to the starting data, thus to ensure the user experience and the original single mode is completely consistent.

\section{Multi-Operating System Deployment Mechanism}

Taking into account the need to deploy multiple operating systems on the local hard disk drive and the need to maintain consistency with the server, the server creates a corresponding cache on the client's hard disk and transmit data in the background by the trickle of technology depending on the multiple operating systems set by the software. Because the client hard disk data and server image data are consistent, when the network is interrupted, the network system and the local system can be realized switching without shutdown by high speed buffer location. As shown in Fig. 1.

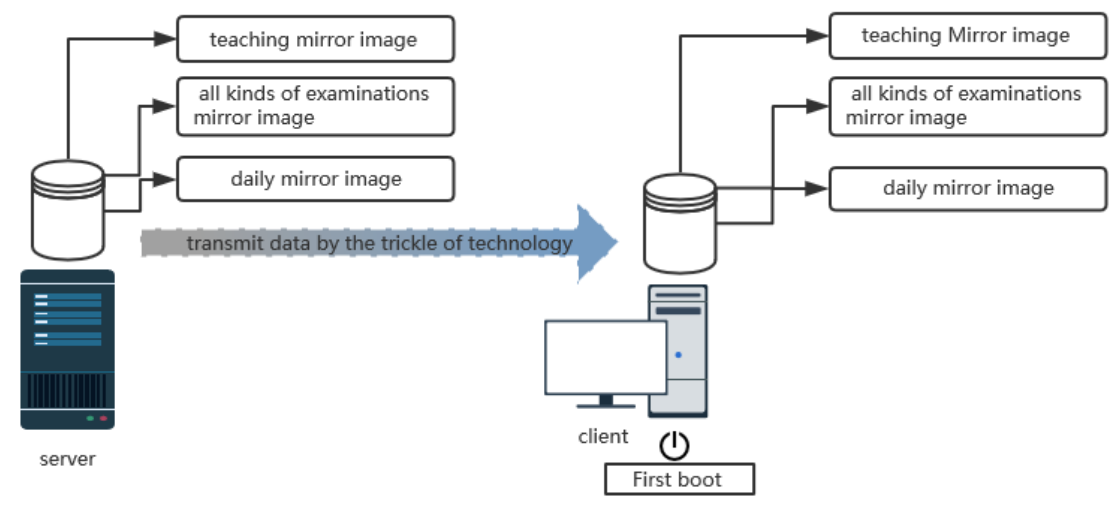

Figure 1. Deployment of multiple operating systems 


\section{Convenient Centralized Management}

(1) Comprehensive centralized management

The laboratory needs different experimental environment. Each computer also needs to install a different operating system or application software. If the administrator install a different environment on computer one by one, it will Not only time-consuming, laborious and inefficient, but also affect the stability of teaching and learning stability. DAWNSTOR desktop virtualization system can centralized manage distributed computer. The use of each student machine can be clearly seen and controlled on the DAWNSTOR server.

(2) Rapid system upgrade

DAWNSTOR desktop virtualization system is working under the network environment. So for Microsoft patch update and the virus database update is very convenient. It can be completed by Room management teacher sitting in his office. All computers will take effect immediately after reboot. Thus, it can greatly improve the computer's security, reduce the spread of computer viruses. This will save a lot of time for network transmission.

(3) Simple maintenance

DAWNSTOR desktop virtualization systems usually do not need to maintenance the client operating system. Even in some special cases, the client is required to deploy the operating system, DAWNSTOR system using the multicast deployment will save more than $60 \%$ of the time than the reduction card.

(4) Support multi operating system

DAWNSTOR desktop virtualization systems need to support multiple operating systems. Administrators will configure the different environment and operating system by the use of different students and different courses.

\section{Diverse User Experience}

(1) Assign virtual desktop by teacher

DAWNSTOR desktop virtualization system can provide classification of the virtual desktop. According to the different application requirements of various courses, it can make different virtual desktop at the server side. After computer room management teacher carry out a simple set in the server, the clients will enter their respective needs of the application environment in the boot, such as the english teaching desktop, the computer basic course special desktop, paperless examination of the desktop, and so on. Each desktop has its own specific application environment and software.

(2) Choose to assign virtual desktop by Student

According to the different needs of various users, DAWNSTOR desktop virtualization system create a variety of application environment. When the students turn on the computer, the system provides the user with a selection menu. Students choose their own application environment. After selection, the system will automatically push the select virtual desktop to the user.

\section{Overall Security}

(1) Remote maintenance and diagnosis

DAWNSTOR desktop virtualization system can perform remote maintenance and diagnosis for client computer. For those simple problems or users of the wrong operation, the management department only need limited management staff to solve it sitting in front of the computer. It greatly shorten the maintenance time and reduce a large number of maintenance personnel than ever before.

(2) Fault tolerant backup

If the operating system cannot be used for normal operation and even collapse due to the software compatibility, virus or wrong operation, it is only necessary to roll back the operating system back to the normal state.

(3) Network security

In the traditional computer room, students' use the Internet or frequent $U$ disk will lead to a large area of computer poisoning. It will result in a breakdown of the system, and the computer cannot be 
used properly. At the start of the student machine every time, DAWNSTOR desktop virtualization system start a clean operating system from the server. It will be a powerful guarantee system security.

(4) Local network dual start

Network boot has a high dependency on the network and the server, if these two failures, it will cause the system to collapse. DAWNSTOR desktop virtualization system with dual boot features can deploy the same operating system as the mirror image on all students through the server. When the network fails, the system will automatically go to the local hard disk drive system to start to ensure the normal operation of the system.

\section{Sound Management Support Services}

(1) Computer behavior management

In the construction of the computer room, the whole system behavior need to be managed. Through setting the black and white list in the soft to prohibit students to use stock software, game software, and video playback, chat software, etc. in a specific time, it can realize the behavior management of students. At the same time, managers can also prevent users from using the USB interface, CD-ROM equipment etc.

(2) The rich management function

In view of the user's usage characteristic, the system provide the rich management function. Such as "Remote switch machine", "one key format", "time synchronization", "shield gateway", "equipment disabled", "software license management", "program management", "user roaming" and other practical functions. It will greatly reduce the maintenance time and maintenance costs of management personnel.

(3) Rapid remote deployment and restore

In the use of the computer room, the system needs to provide a variety of operating system restore mode, and to realize the fast remote deployment of the operating system. It can deploy operating system for the students' computer in 10 minutes.

\section{Strong Environmental Adaptability}

(1) Perfect network adaptability

Desktop virtualization system can use a variety of network environment and can support the network environment across the VLAN. In the case of no adjustment of the switch parameters and even the student machine without the network boot option, it can achieve the client's cross VLAN start by using the international original DAWNSTOR--Boot start technology.

(2) Computer hardware independence

No matter what kind of machine or brand, the students can use the same operating system to start. It not only solve the hidden dangers of the school client as a result of damage to the replacement of different hardware and that restore the card cannot be used, but also solve the puzzle that need to back up a variety of different drivers, version

\section{Strong Environmental Adaptability}

(1) Perfect network adaptability

Desktop virtualization system can use a variety of network environment and can support the network environment across the VLAN. In the case of no adjustment of the switch parameters and even the student machine without the network boot option, it can achieve the client's cross VLAN start by using the international original DAWNSTOR--Boot start technology.

(2) Computer hardware independence

No matter what kind of machine or brand, the students can use the same operating system to start. It not only solve the hidden dangers of the school client as a result of damage to the replacement of different hardware and that restore the card cannot be used, but also solve the puzzle that need to back up a variety of different drivers, version. 


\section{Networking Solutions}

At present, our computer room network is a separate network of each computer room connected through 1 network lines. On the same campus of different teaching building room, this paper adopts the following scheme: Different teaching building of a number of laboratory room are communicated with a core switch. Core switch is divided into VLAN to manage network for multiple laboratory. Several computer rooms use 2 dedicated management server. As shown in Fig. 2.

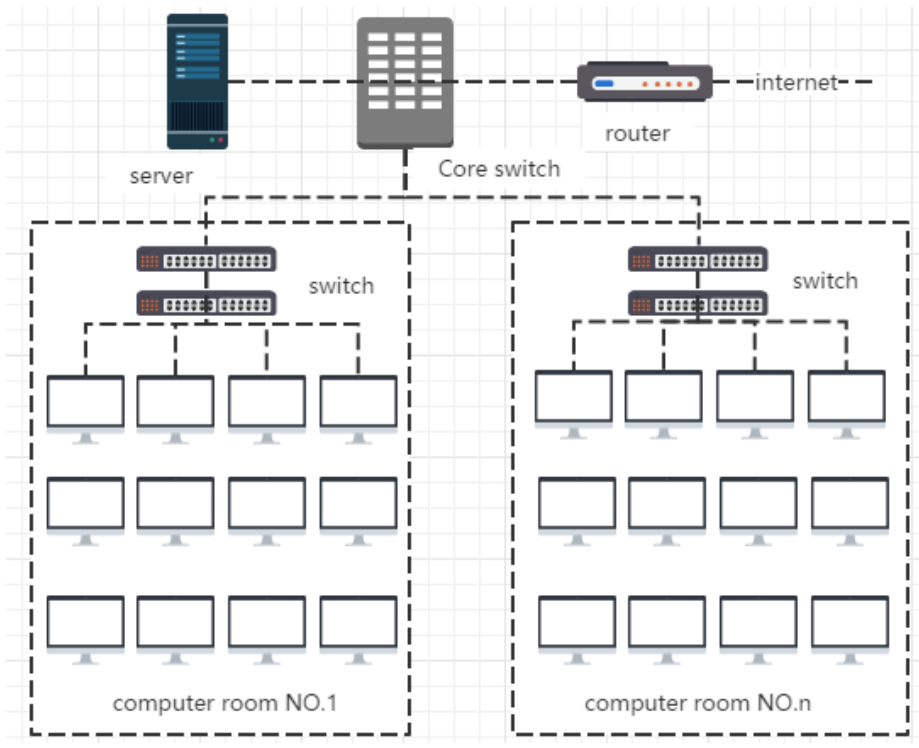

Figure 2. Multi-computer room mode

\section{The Whole Experiment Scheme of Virtualization}

The overall experimental scheme is shown in Fig. 3.

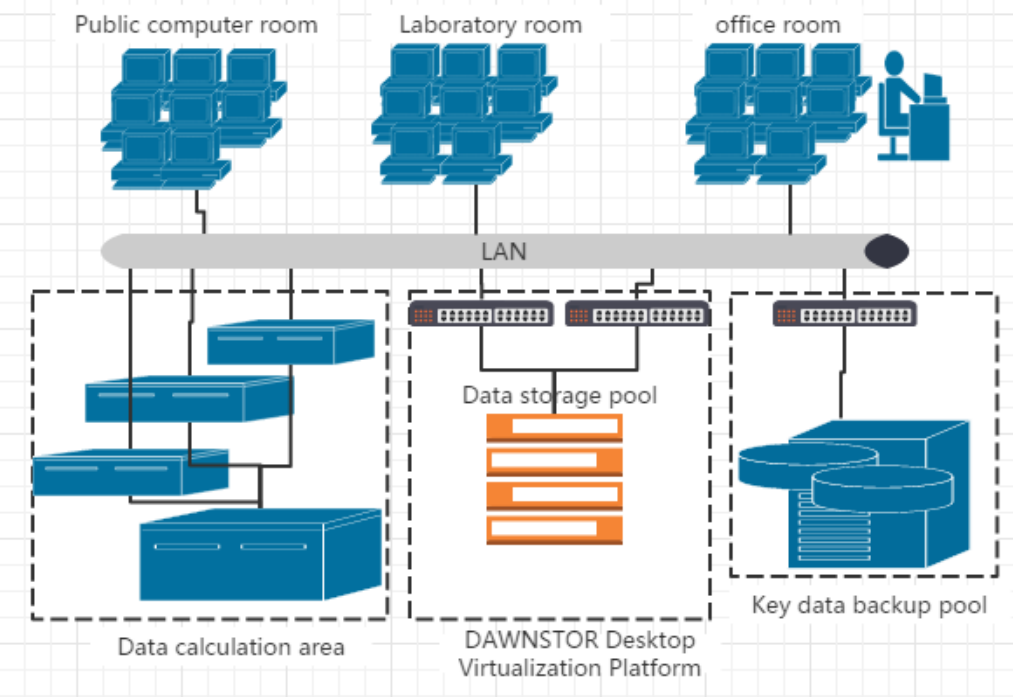

Figure 3. The overall experimental program

\section{Desktop Virtualization Management for Multiple Computer Rooms}

Multiple computer rooms use more than one dedicated management server to manage with easy and safe group mode. In order to avoid the single point of failure of the server, the scheme is based on the establishment of a data storage pool. DAWNSTOR desktop virtualization software system image database storage pool can effectively protect data security. The data storage pool can take over the 
service, restore the image data and ensure the normal operation of the computer room when the server is in trouble because of the accident.

\section{Data Backup}

Data backup platform meet the needs of efficient data backup and recovery to add a layer of security to the desktop virtualization platform.

\section{Computer Laboratory Room Implementation Plan}

\section{Virtualization Local Cache}

(1) Local hard disk format (for a new computer, without formatting, bare metal can).

(2) Start at the first time

In the first start, the server will pushed the system data on the server automatically to the client on the hard disk using ON-Demand technology. Client hard disk at the same time storage client data and the system of temporary files to form cache.

(3) Start later

Later, each time you start the client, server DAWNSTOR only transfer a small amount of I / O read and write data, this part of the data and cache data combined become the start of the data, which is identical to the user experience and the original single mode. When a user needs to use more data in the process of using the client computer with pushing the corresponding data from the server to the client's hard disk.

\section{Deploy Multiple Operating Systems}

Taking into account the local hard disk drive needs to deploy multiple operating systems and to maintain consistency to servers, according to the DAWNSTOR set of multiple operating systems, the server create $t$ the corresponding cache on the hard disk of client and transmit data in the background with the trickle of technology. When the network is interrupted, the network system and the local system can be realized to switch without shutdown with high speed cache because of the consistency of the client hard disk data and the server image data.

\section{Virtual Desktop Image}

1) Basic mirror

When the system is installed for the first time, it is required to find a client to install the operating system and the basic operation software. After the installation is completed, the operating system is posted to the server to form a basic image.

2) Incremental snapshot image

After the manager added a part of the software, the snapshot of the incremental image is formed on the server. This image is composed of two parts: the basic image and the incremental part. So the user can switch between the basic mirror and the incremental part.

3) Logical mirror

When new software is added, the combination of the basic mirror image and the incremental snapshot will form a logical image, and the rest of the client can use the newly installed software automatically as long as the logical mirror image is selected.

4) New basic image

When the incremental snapshot is more and more, you can merge the basic mirror image and the incremental snapshot image, thus forming a new basic image.

\section{Push on Demand}

There are many application system images on the virtual server. When the client is turned on, the server will distribute the private application system and desktop for the client based on the different account of the client's landing. The personalized needs of each class and each student can be pushed. 


\section{Conclusions}

This paper discusses the construction program by use of DAWNSTOR system to build a virtual cloud desktop system quickly and efficiently in the laboratory room. The purpose is to solve the problems in the construction investment, management and maintenance and operation of the laboratory computer room in our university, and to achieve standardized management of the computer room system, data centralized control, to eliminate the virus and reduce the total cost. By using the desktop virtualization, we can also serve as a computer laboratory, at the same time as a variety of computer teaching and social examination and training, to meet the needs of all applications on the computer room system environment. At the same time, it also ensures the system environment of the students' system, such as the free machine, the individualized instruction, the examination, the outside training and so on. The scheme can effectively improve the utilization rate of teaching facilities, realize the free switching of multi system and diversified teaching environment, reduce the management pressure of the management teacher to the computer room, and improve the experimental teaching level.

\section{Acknowledgment}

This work is supported by the project of the basic scientific research business of Beijing University of Chinese Medicine (No. 2015-JYB-JSMS052), all support is gratefully acknowledged.

\section{References}

[1] "Virtualization and cloud computing" group. Virtualization and cloud computing [M]. Beijing: Publishing House of electronics industry, 2009, 48-49

[2] Wikipedia. Desktop_virtualization [EB/OL]. http://en.wikipedia.org/wiki/Desktop_virtualization: Wikipedia, 2012. 